\title{
Remeasurement of a Silicon Lattice Period
}

\author{
RICHARD D. DESLATTES, MITSURU TANAKA, GEOFFREY L. GREENE, ALBERT HENINS, AND \\ ERNEST G. KESSLER, JR.
}

\begin{abstract}
Opitcal interferometry of an Silicon lattice period is an important link between macroscopic and microscopic lengths as well as between low-energy and high-energy spectroscopies. An evident discrepancy between two pre-1982 measurements has limited the effective application of these results. Very recent results, reported here in a preliminary way, appear to further understanding and removing this discrepancy.
\end{abstract}

\section{BACKGROUND AND Motivation}

$\mathrm{M}$ EASUREMENTS linking local networks of secondary standards occurring in different spectral regions are important throughout the entire electromagnetic spectrum. The story is particularly well known to this conference in the case of the microwave-to-optical connection where the realization of a frequency synthesis chain, together with the availability of narrow-line optical oscillators, has permitted effective unification of these two regions. The situation arising when one attempts to continue this progession to the $X$ - and $\gamma$-ray regions is perhaps less widely known but presents also some serious challenges and offers, as well, interesting rewards for successfully overcoming the evident difficulties. Although there are procedures involving many relatively small steps through the UV- and X-ray region these are not capable of giving accurate results largely because of the absence of narrowband oscillators in the intermediate (X-ray) region. To some extent, perfect monocrystalline lattices offer an alternative to sharp lines, at least in the region between 0.1 and $1.0 \mathrm{~nm}$, but these are not fully equivalent to narrowline oscillators even over the limited band in which they are available. Once one reaches the region of intense nuclear $\gamma$-rays (ca $100 \mathrm{keV}$ and above), sharp lines are again available but the problem of linking these to visible light is formidable.

In recent years a practical procedure has emerged for covering the 18 octaves between red light and $400 \mathrm{keV}$ (the local standard lamp for $\gamma$-ray people). In the first step of this procedure, the lattice period of a perfect monocrystalline silicon slab is determined by simultaneous $\mathrm{X}$ ray and optical interferometry of a common baseline. A

Manuscript received June 23, 1986

R. D. Deslattes, G. L. Greene, A. Henins, and E. G. Kessler, Jr. are with the Quantum Metrology Group, National Bureau of Standards, Gaithersburg, MD 20899.

M. Tanaka was with the Quantum Metrology Group, National Bureau of Standards, Gaithersburg, MD 20899. He is now with the Nano-Metrology Group, Quantum Metrology Division, National Research Laboratory of Metrology, 1-chome, Umezono, Sakura-mura, Niihari-gun, Ibarki 305, Japan.

IEEE Log Number 8613467 second step transfers this lattice calibration to other specimens of germanium, silicon, or $\mathrm{SiO}_{2}$ whose morphology is suited to $\gamma$-ray diffraction. These calibrated specimens are then used in an angle-measuring machine capable of internal calibration to measure the $\gamma$-ray wavelengths by means of the Bragg-Laue $\lambda=2 d \sin \theta$, which is exact in the symmetric transmission geometry. Results already obtained, or obtainable, through this procedure are of more than casual significance for determinations of fundamental constants, tests of basic theory, and establishing the masses of elementary particles. For example, measurement of the 220 repeat distance in silicon, $d_{220}$, establishes the cell edge dimension, $a_{0}=d_{220} \sqrt{8}$ and unit cell volume $a_{0}^{3}$. When this is combined with a (sample specific) mean molar mass $A$ and a measured density $\rho$ one obtains a value for the Avogadro constant, $N_{A}=$ $n A / \rho a_{0}^{3}$ where $n=8$ is the number of atoms in each unit cell [1]. Besides this, one can measure optical transitions in one- and two-electron ions and muonic atoms to test basic theory. One can also measure Rydberg transitions in other exotic atoms to determine meson masses, specifically, those of the negative pion and the negative kaon [2]; the $n+p$ capture $\gamma$ can be interpreted as yielding the neutron mass [3]. Finally, when the scale is extended to sufficiently high energies that the corresponding mass change can also be measured with high relative accuracy, a new combination of fundamental constants, namely $N_{A} h / c$, becomes available for increasing the order of overdetermination in adjustments of the fundamental constants and for enforcing consistency between the local scale for high-energy $\gamma$-rays and that for low-energy $\gamma$ rays [4].

Regardless of subsequent further steps, the first step in all these chains is the X-ray/optical interferometer (XROI) exercise. Two laboratories reported results from such exercises before 1982 [5]-[7]. The first result came from our group at the National Bureau of Standards (NBS) using optical Fabry-Perot interferometry while the second came from Physikalisch-Technische Bundesanstalt (PTB) using a two-beam polarization interferometry of the sort developed by Curtis et al. [8]. Results reported by these groups differ by $1.8 \mathrm{ppm}$, an amount large compared with the joint standard deviation $(0.35 \mathrm{ppm})$ and likely sample-tosample variations. After two years' contemplation of this situation, it seemed likely that the measurements were discrepant with at least one wrong. Accordingly, we set about to reestablish the XROI experiment at NBS with several significant technical improvements and to repeat 
in this improved environment the NBS measurements last carried out in 1974. The remainder of this paper briefly describes the improved NBS XROI experiment, the performance of a new lattice comparator (delta-d machine) and reports preliminary data which indicate that the earlier NBS result was wrong.

\section{Features of the Improved NBS XROI EXPERIMENT}

Looking back to the early NBS measurement, several weaknesses are evident. First, only a fixed-frequency $\left(I_{2}\right.$ stabilized) laser was available. With the Fabry-Perot optical interferometer (see Fig. 1(a)) locked to the laser, the only measurable feature was the $\mathrm{X}$-ray counting rate. This was turned into a phase value by noting average counting rate and (X-ray) fringe contrast. Second, the two components of path curvature had to be treated in different ways, neither of which was both precise and available during the measurement. Components of trajectory error are usually referred to as pitch, roll, and yaw; the interferometer is rather insensitive to roll error. In the case of yaw error (see Fig. 1(b)) the offset, $Z(\approx 3 \mathrm{~mm}$ ) was determined by dimensional measurement while the (approximately uniform) path curvature was determined in a separate (X-ray) measurement of the rotation $\delta \Theta$ in the "standard" displacement; this $z \delta \Theta$ correction amounted to $0.35 \mathrm{ppm}$. In the case of "pitch" error, the offset $y$ was nominally zero and assumed to be $\pm 0.5 \mathrm{~mm}$ while the rotation $\delta \rho$ was estimated to be less than 0.2 nanoradian from the constancy of $\mathrm{X}$-ray fringe contrast over the customary displacement.

In the new version of this experiment, specific steps have been undertaken to secure both a more sensitive and precise determination of X-ray phase values and to provide for on-line measurement of trajectory errors. To determine X-ray phase values more precisely, we introduced a frequency agile local oscillator laser which could be stepped over a sufficiently wide range of frequencies $(\approx$ $300 \mathrm{MHz}$ ) that the system scanned an entire X-ray period. The offset frequencies were actively stabilized and data such as are shown in Fig. 2(a) obtained. To measure path curvature we introduced a four-beam interferometer of the polarization-encoded type whose signals could be processed to yield tilt angles independent of displacement over a reasonable range of linear motion. To directly evaluate the effect of curvature on X-ray phase, i.e., to measure the Abbe offset distances, we introduced deliberate pitch and yaw motions and noted the $\mathrm{X}$-ray phase changes induced thereby. The results are shown in Fig. 2(b) for yaw and Fig. 2(c) for pitch. The steep monotonic data in Fig. 2(b) reflects the known large horizontal offset and confirmed in a general way the early $\mathrm{X}$-ray measurements of path curvature. The pitch results shown in Fig. 2(c) are seen to have various slopes depending on the relative position of the X-ray and optical beam centroids. By careful setting of this degree of freedom, the coefficient can be made close to zero or it can be allowed to differ from zero and the appropriate correction applied. In either case, the

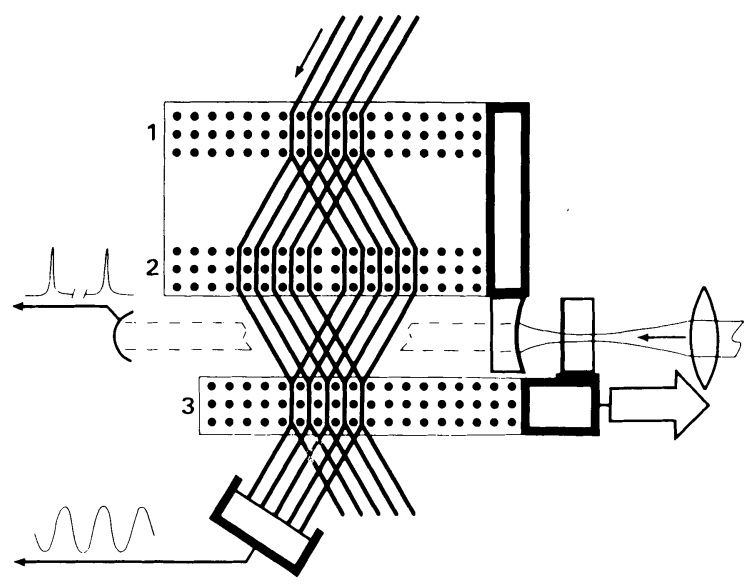

(a)

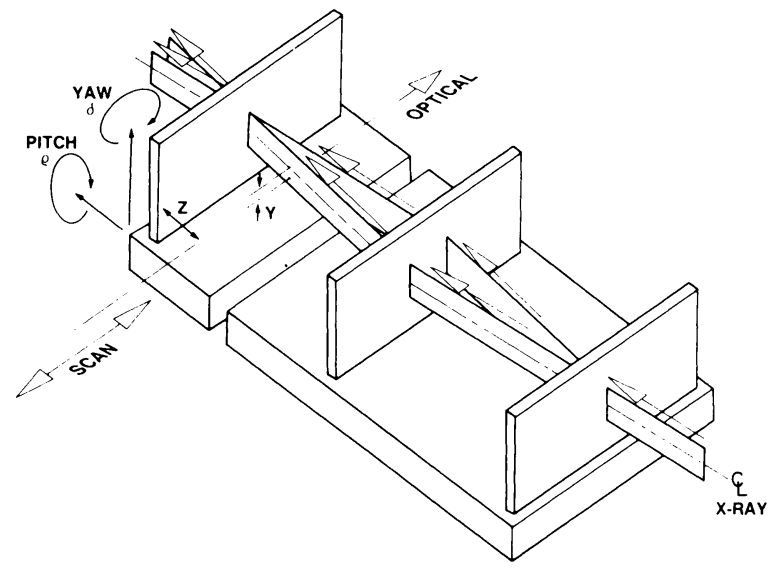

(b)

Fig. 1. Diagrams of an X-ray/optical interferometer (XROI) measurement: (a) The principle; (b) the main motion errors which need to be evaluated.

data runs themselves show that there is a greater pitch error than had been estimated previously by fringe contrast stability and that previously used geometrical alignment procedures could easily result in offsets greater than $1 \mathrm{~mm}$. These two tendencies acting in concert can easily produce mistakes greater than $1 \mathrm{ppm}$.

At the present time, the new system appears to operate well and to be giving results 1.6 to $1.7 \mathrm{ppm}$ smaller than those previously obtained. Sufficient data have not yet been obtained and our analysis is at an early stage. However, it would seem that results having an estimated standard deviation below $0.1 \mathrm{ppm}$ are at hand. Actually lattice values obtained in an overnight run give statistical imprecision near $0.02 \mathrm{ppm}$. Estimates of the influence of other error terms, e.g., temperature uncertainty and the curvature terms are not yet complete but appear comparable. Nevertheless, when all is finished in this measurement its result applies only to a local region of the particular crystal sample currently in the apparatus which leads us to the next section of this report.

\section{A New Lattice Comparator}

The difficulty of XROI measurements and the need for diffraction crystals whose size and shape make them un- 


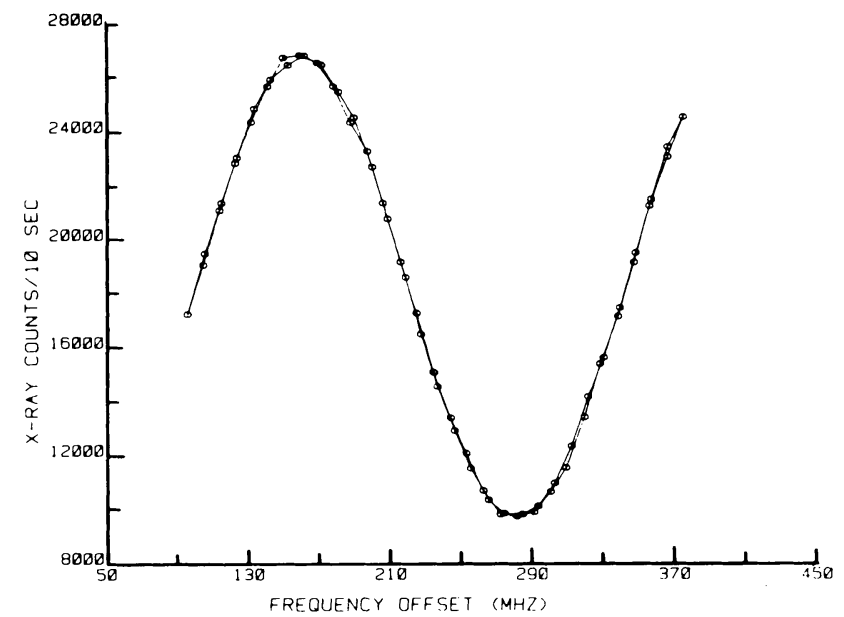

(a)

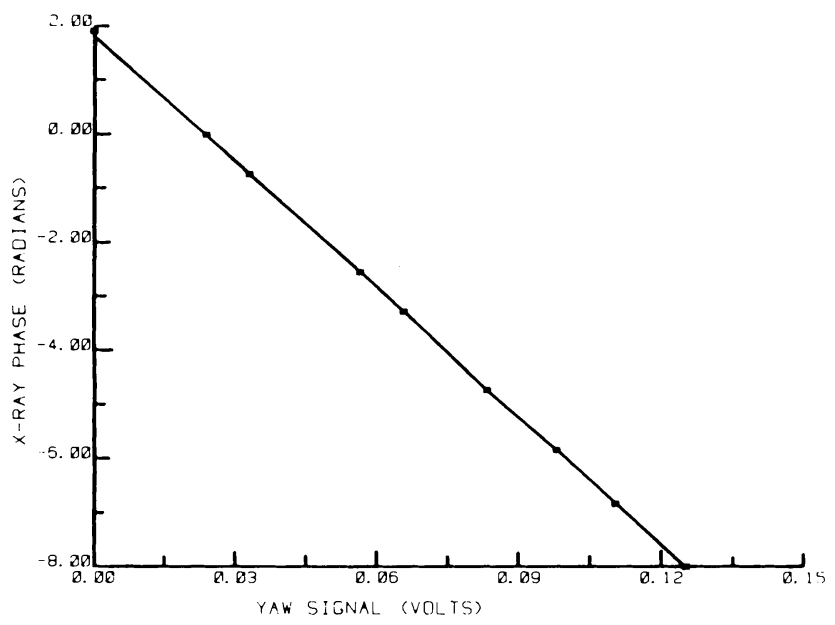

(b)

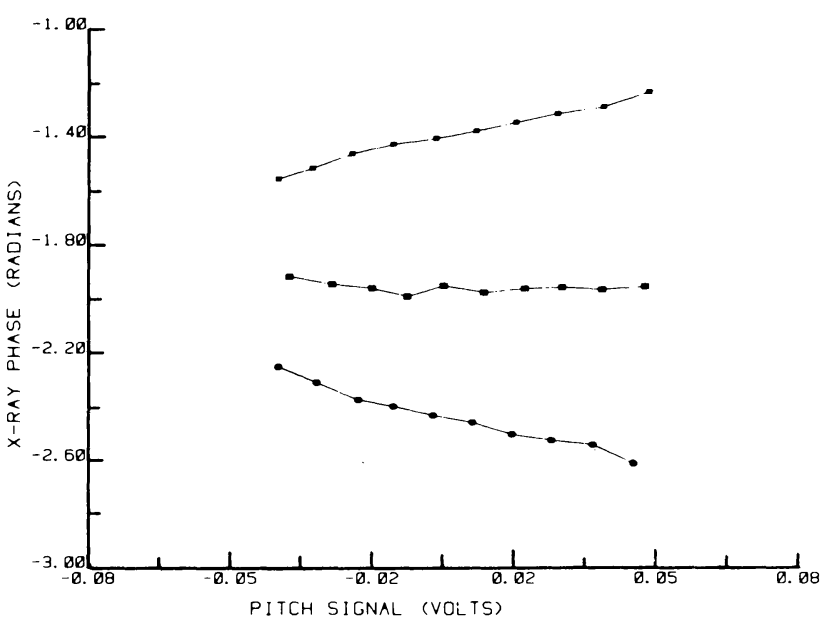

(c)

Fig. 2. Samples of data from XROI. (a) Data from which X-ray phase is extracted. (b) Evaluation of the yaw coefficient from $X$-ray phase changes; the large slope corresponds to the $3-\mathrm{mm}$ offset between X-ray and optical paths. (c) Various pitch coefficients corresponding to small changes in the relative position of $\mathrm{X}$-ray and optical centroids which can be made closely coincident as in the center curve.

suited to X-ray interferometry make it desirable to have a convenient rapid method to compare specimens of the same material and of different materials as well. The essential features of the needed procedure were introduced

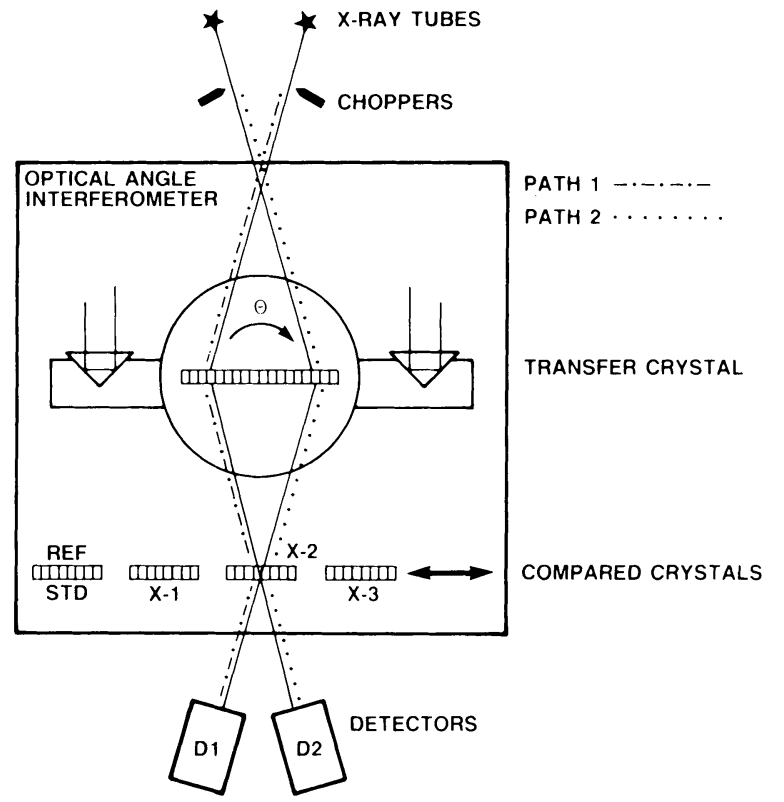

(a)

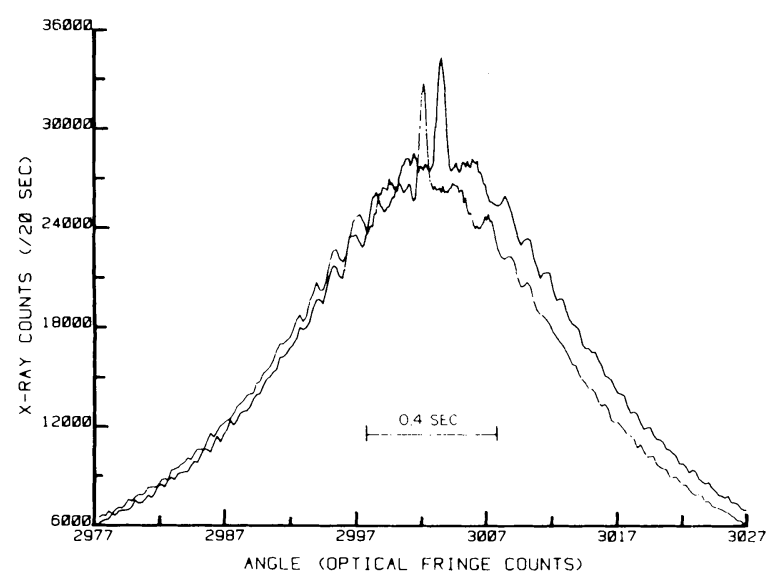

(b)

Fig. 3. (a) Schematic of lattice comparison apparatus. (b) Typical profiles with important fine detail useful for precise comparison of crystal spacings.

by Hart [9] and an apparatus based on these principles is currently in use at the PTB [10]. We have built and now operate a somewhat more elaborate system having precision and accuracy together with the ability to automatically exchange several samples.

The main features of this nondispersive procedure for lattice parameter comparison are indicated in Fig. 3(a). Radiation from sources 1 and 2 alternatively proceeds along paths 1 and 2 to the detectors shown. If the long crystal were straight, uniform, and had the same lattice period as the particular sample in place, then the diffraction profiles registered by the two detectors as the long crystal is rotated would coicide. These assumptions about the long crystal are generally wrong so that the curves will not generally conincide; such an offset is of no consequence since our objective is to compare the small crystals with one another. Should a pair of these have, perchance, identical lattice parameters, then their offsets 
would match. Differing offsets would signal differing lattice parameters according to $\delta d / d=\delta \theta / \tan \theta$.

Certain special problems and opportunities are associated with this approach to lattice comparisons. First, the two paths indicated in Fig. 3(a) are not useable simultaneously since the forward-diffracted plus transmitted beams from the unwanted ray path would swamp the detector. This requires a system of shutters or choppers to define the path being used. Second, the fine detail seen in Fig. 3(b) has important consequences. When it can be studied reliably it provides a basis for considerable gain in precision compared to cases where only broader smooth distributions are discernable. Conversely, when such structures are present and not clearly resolved, serious systematic difficulties can be encountered, as surely was the case in some of our early work on crystal-to-crystal transfer measurements [2].

To take maximum advantage of potential gain in sensitivity and to provide for a variety of related measurements, our new apparatus has been fitted for wide-range interferometric angle measurement. The dual-source spectrometer is, in fact, under continuous interferometric control over its entire range of $\pm 20^{\circ}$. Examples of the results currently being obtained with this system are shown in Fig. 3(b). As noted above, the general offset is characteristic of the first crystal's nonuniformity and its difference from the second. However, changes in this picture between pairs of small crystals are entirely characteristic of the difference between the two samples probed. Visual inspection is already sufficient to see changes of the order of $0.1 \mathrm{ppm}$ while, with a modest level of analysis, $0.01 \mathrm{ppm}$ comparisons are in reach. Since the detailed shapes are calculable from dynamic diffraction theory a considerable further improvement appears practical. There is also a quite convenient correlation integral procedure which avoids entirely the need for detailed modeling of the profiles [11].

\section{Conclusion and Outlook}

New and more refined capabilities for X-ray/optical interferometry (XROI) and intercomparison of crystal re- peat distances have been established at NBS. These are operating at levels below $0.1 \mathrm{ppm}$ and appear to require little further development in order to reach even better precision and possibly accuracy as well. Early results from the XROI experiment have revealed the presence of a significant systematic problem with the previous NBS result. The new values appear to eliminate the large discrepancy between the results from PTB and the previous NBS value. When a fully closed-loop is completed by use of the new lattice comparator (delta- $d$ machine) it should be clear what level of interlaboratory consistency has been achieved at the present state of development in this still relatively new technology.

\section{REFERENCES}

[1] P. Seyfried et al., "The Avogadro constant-Recent results on the molar volume of silicon," "pp. 161-165, this issue.

[2] R. D. Deslattes, E. G. Kessler, W. C. Sauder, and A. Henins, "Remeasurement of $\gamma$-ray reference lines," Ann. Phys., vol. 129, no. 2, pp. 378-434, Oct. 1980.

[3] G. L. Greene, E. G. Kessler, Jr., R. D. Deslattes, and H. Börner, "New determination of the deuteron binding energy and the neutron mass," Phys. Rev. Lett., vol. 56, no. 8, pp. 819-822, Feb. 1986.

[4] R. D. Deslattes, G. L. Greene, and E. G. Kessler, Jr., "Absolute prompt gamma-ray spectroscopy and the determination of fundamental constants," J. Phys., vol. 45, no. 3, pp. C3-41-46, Mar. 1984.

[5] R. D. Deslattes, "Application of x-ray interferometry," in Precision Measurement and Fundamental Constants II, B. N. Taylor and W. D. Phillips, Eds., Nat. Bur. Stand., Spec. Publ. no. 617, 1984, pp. 303-311.

[6] P. Becker, et al., "Absolute measurement of the (220) lattice plane spacing in a silicon crystal," Phys. Rev. Lett., vol. 46, no. 23, pp. 1540-1543, June 1981.

[7] P. Becker, P. Seyfried and H. Siegert, "The lattice parameter of highly pure silicon single crystals," $Z$. Phys. B, vol. 48, pp. 17-21, 1982.

[8] I. Curtis, I. Morgan, M. Hart and A. D. Milne, "A new determination of Avogadro's number," in Precision Measurement and Fundamental Constants, D. N. Langenberg and B. N. Taylor, Eds., Nat. Bur. Stand., Spec. Publ. no. 343, 1971, pp. 285-289.

[9] M. Hart, "High precision lattice parameter measurements by multiple bragg reflexion diffractometry," in Proc. R. Soc. London A, vol. 309, no. 1497, Mar. 1969, pp. 281-296.

[10] H. Siegert, P. Becker and P. Seyfried, "Determination of silicon unit cell parameters by precision measurement of bragg plane spacings," Z. Phys. B, vol. 56, pp. 273-278, 1984.

[11] D. Windisch, "Präzisionsvergleich von $\{100\}$-netzebenen-abstanden in silicium-einkristallen," Physikalisch-Technische Bundesanstalt, Braunschweig, PTB Bericht APh-27, Jan. 1986, to be pubished. 